\title{
A Case Report of Progressive Multifocal Leukoencephalopathy in Peritoneal Dialysis
}

\author{
Lilia Ben Lasfar ${ }^{\mathrm{a}, \mathrm{c}}$, Yosra Guedria, Sinda Mrabet ${ }^{\mathrm{a}}$, Dorsaf Zellama ${ }^{\mathrm{a}}$, Anis Ben Hassine ${ }^{\mathrm{b}}$, \\ Wissal Sahtout ${ }^{\mathrm{a}}$, Awatef Azzebia ${ }^{\mathrm{a}}$, Salma Toumi ${ }^{\mathrm{a}}$, Asma Fradia, \\ Sofiene Ben Ammou ${ }^{\mathrm{b}}$, Abdellatif Achour ${ }^{\mathrm{a}}$
}

\begin{abstract}
We experienced a case manifesting progressive multifocal leucoencephalopathy (PML) in peritoneal dialysis (PD). A 27-year-old male patient had received a chronic PD therapy for 4 years. He had a past medical history of hypertension and myelodysplastic syndrome. $\mathrm{He}$ complained of hemiparesis with sudden onset and behavioral disorders. The patient seemed indifferent, incoherent with deficit walking. The cranial nerve examination showed a left central facial paralysis. Brain CT scan showed a paramedian right low density area not systemized, located in the corpus callosum and the centrum semiovale. Brain MRI confirmed the presence of PML by the detection of signal abnormalities in bilateral and asymmetrical white matter. The culture of cerebral spinal fluid was negative. The patient underwent six sessions of plasma exchange with favorable evolution. Few cases of PML have been reported in hemodialysis patients and no case has been previously described in PD. In our case, under immunocompromised conditions, precipitating factors appear multifactorial. Depressed immune system induced by chronic dialysis as well as liver disease and myelodysplasia might contribute to the development of florid clinical manifestation of PML.
\end{abstract}

Keywords: Progressive multifocal leukoencephalopathy; Peritoneal dialysis

\section{Introduction}

Progressive multifocal leucoencephalopathy (PML) is an opportunistic, demyelinating, neurological disorder caused frequently and not necessarily by an ubiquitous polyomavirus, called JCV [1,2]. Generally, it is characterized by multifocal

Manuscript submitted August 25, 2017, accepted October 6, 2017

${ }^{a}$ Department of Nephrology, Dialysis and Kidney Transplantation, Sahloul Hospital, Sousse, Tunisia

bepartment of Neurology, Sahloul Hospital, Sousse, Tunisia

${ }^{\mathrm{c} C}$ Corresponding Author: Lilia Ben Lasfar, Department of Nephrology, Dialysis and Kidney Transplantation, Sahloul Hospital, Sousse, Tunisia.

Email: lililasfar@gmail.com

doi: https://doi.org/10.14740/wjnu316w or monofocal asymmetric subcortical lesions in the white matter. It can affect cortical gray matter [1].

The first case was described in a patient with chronic lymphocytic leukemia. The incidence of PML has rapidly increased, in the AIDS era [3].

PML occurs in patients with immunosuppression due to leukemia or lymphomas or cancer chemotherapy or immunosuppressive therapy or chronic inflammatory disease. Next to these rare immunocompromised diseases, there have been reports showing the development of PML in chronic hemodialysis [4].

Few cases have been reported in hemodialysis [5], but no cases were reported in peritoneal dialysis (PD).

Usually, the clinical outcome of patients with PML is unfavorable with progression to death within 6 months after onset of symptoms [2].

In absence of specific therapy, 7-9\% of patients with PML demonstrate prolonged survival ( $\geq 12$ months).

In the present paper, we report a case of PML in PD associated with chronic liver disease and myelodysplasia during chronic PD.

\section{Case Report}

A 27-year-old man had been on PD therapy for 4 years for end-stage renal disease caused by chronic interstitial nephritis. He had a family history of renal failure in sister and maternal aunt and a personal history of metabolic inherited disease diagnosed in pediatrics at the age of 5 years because of recurrent hypoglycemia and ketosis.

He was diagnosed with renal failure, hypertension and chronic liver disease in July 2011 at the age of 22 years. Serum creatinine was $429 \mu \mathrm{mol} / \mathrm{L}$. Abdominal ultrasound showed a normal sized liver, with dysmorphic atrophy in the fourth segment and signs of portal hypertension with a splenomegaly $(14 \mathrm{~cm})$, and echostructure was homogeneous without focal lesion.

The biological exploration for the liver disease showed negative serology for hepatitis $\mathrm{C}$ and $\mathrm{B}$. Immunological exams showed negative antinuclear, anti-smooth muscle, anti-mitochondria and anti-LKM1 antibodies. Similarly, the serological markers of celiac disease were negative (anti-deamidated gliadin peptides, anti-endomysium, and anti-transglutaminase an- 

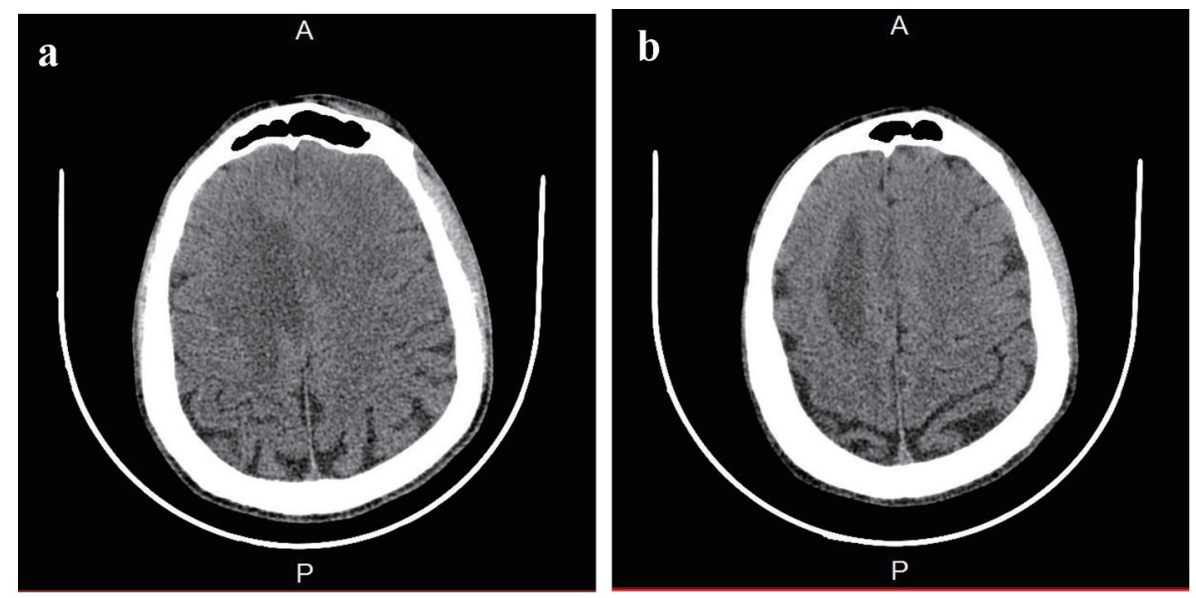

Figure 1. Brain CT. (a) A paramedian right low density area located in the corpus callosum and in the white matter of the right centrum semiovale. (b) A hypodense zone with a discrete mass effect on the right ventricle.

tibodies). The liver biopsy confirmed the presence of discrete intrahepatocyte cholestasis without storage disease.

Sternal puncture was performed to explore a macrocytic anemia (hemoglobin $(\mathrm{Hb}) 7 \mathrm{~g} / \mathrm{dL}$ ) and a thrombocytopenia $\left(60,000\right.$ platelets $\left./ \mathrm{mm}^{3}\right)$. The myelogram showed average wealth of marrow with presence of megakaryocytes and signs of dyserythropoiesis. Therefore, anemia is megaloblastic and the origin of thrombocytopenia is peripheric.

He experienced no other complications, when he began to complain of behavioral disorders and was diagnosed as having left hemiparesis with sudden onset without visual disturbances or headaches or vomiting. His blood pressure was 190/100 mm $\mathrm{Hg}$. Respiratory rate was 23 breaths/min and the cardiopulmonary examination was without any abnormalities. Neurological examination showed a normal consciousness level, deficit walking. Voluntary motor system examination showed early fall of the left upper limb. Masseter, biceps and triceps tendon, and knee tendon reflexes were present, but brisk in knee tendons, plantar reflexes were indifferent. There was no dysmetria in finger-to-nose test nor adiadochokinesia. The cranial nerve examination revealed a left central facial. Sensory system was normal. The major abnormal findings were as follows: $\mathrm{Hb} 7 \mathrm{~g} /$ dL, white blood cell count $3,090 / \mathrm{mm}^{3}$, platelets count 84,000 / $\mathrm{mm}^{3}$, serum creatinine $1,147 \mu \mathrm{mol} / \mathrm{L}$.

Serological examination for viral infection including HIV, herpes simplex virus and cytomegalovirus showed negative findings. Lyme disease was eliminated (serology was negative). Immunological examination showed negative antinuclear antibodies and anti-cytoplasm neutrophil polynuclear.

Brain CT scan showed a paramedian right low density area not systemized, located in the corpus callosum and in the white matter of the right centrum semiovale, a discrete mass effect on the right ventricle, unexpanded ventricular system and no stigma of bleeding (Fig. 1). Brain MRI confirmed the presence of PML by the detection of signal abnormality in periventricular, frontal, cortical, subparietal white matter, right centrum semiovale knee and corpus callosum. It was flair hyperintense, moderate enhancement especially at the knee of the corpus
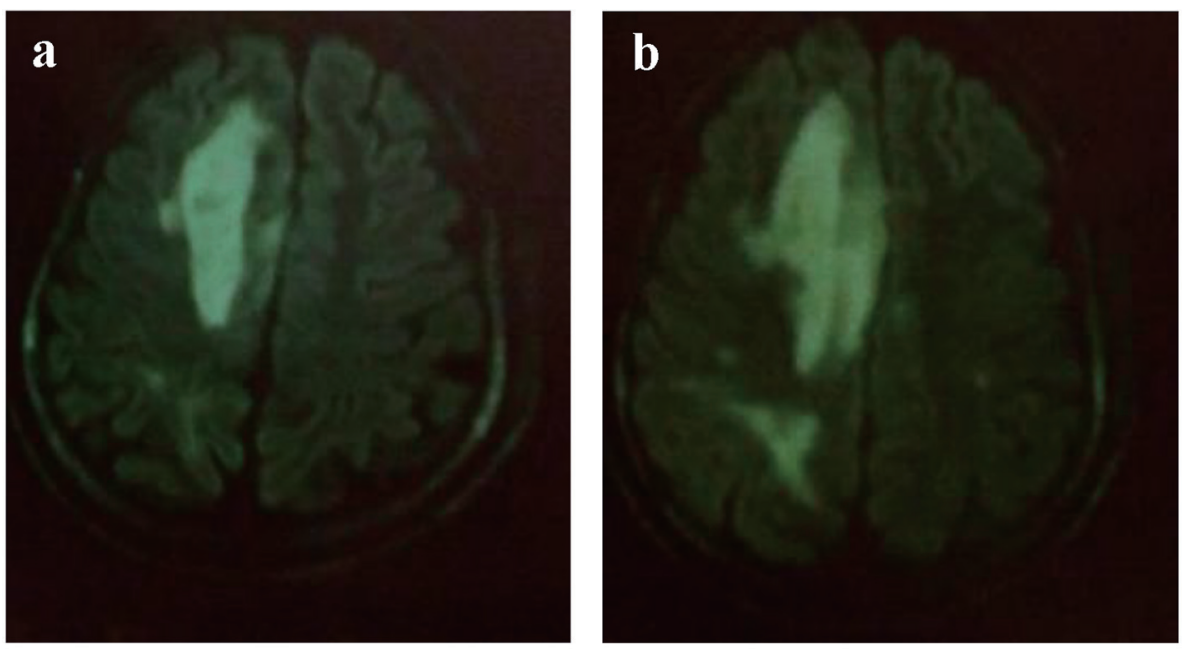

Figure 2. (a, b) MRI: cerebral axial section: Flair hyperintense areas periventricular, frontal, cortical, subparietal white matter, right centrum semiovale knee and corpus callosum (bilateral and asymmetric areas) evocated PML. 
callosum and in the right oval center, without mass effect on other structures. These signal abnormalities were detected in bilateral and asymmetric areas. So, it evocated first the PML lesions (Figs. 2 and 3).

The patient's cerebral spinal fluid (CSF) was normal pressure, hemorrhagic and xanthochromic in appearance. The CSF contained normal white cell counts $\left(\leq 1 \mathrm{~mm}^{3}\right)$, and high erythrocytes count $\left(2,500 / \mathrm{mm}^{3}\right)$, normal protein concentration $(1.3$ $\mathrm{g} / \mathrm{dL})$ and normal sugar concentration $(2.9 \mathrm{mmol} / \mathrm{L})$.

CSF examination showed negative findings for bacterial culture, but rearranged regulatory region of JCV DNA was negative detected with the use of a nested polymerase chain reaction. No oligoclonal IgG band or elevated myelin basic protein was found.

The patient had six sessions of plasmapheresis with partial improvement (partial disappearance of hemiparesis).

During plasma exchanges, enterococcus faecalis septicemia had occurred. This required the use of intravenous antibiotics: penicilline A and ciprofloxacine PerOs.

Initially, the evolution was favorable with complete recovery until 3 months. Secondary, there was a relapse with aggravation of the memory deficiency and amputation of the visual field. The symptoms have progressed rapidly (2 days). A neurological examination was done during this recurrence, and there were a temporospacial disorientation, a decreased verbal fluency, right homonymous lateral hemianopia and pyramidal syndrome without motor deficit. During investigations, serology (herpes/hepatitis B and C) was negative, PCR (BKV and $\mathrm{JCV}$ ) and HSV in plasma were negative, and anti-conoronal antibodies and tumor markers were negative too. In the CSF, protein concentration was $1 \mathrm{~g} / \mathrm{dL}$ without leucocytes with clear fluid and PCR JCV in the CSF was negative. At imaging, brain CT showed an expansive hypodensity in left cortico and under cortical parieto-occipital area and in the white matter of the right centrum semiovale (Fig. 4). The MRI showed an important regression of the first demyelinating range previously

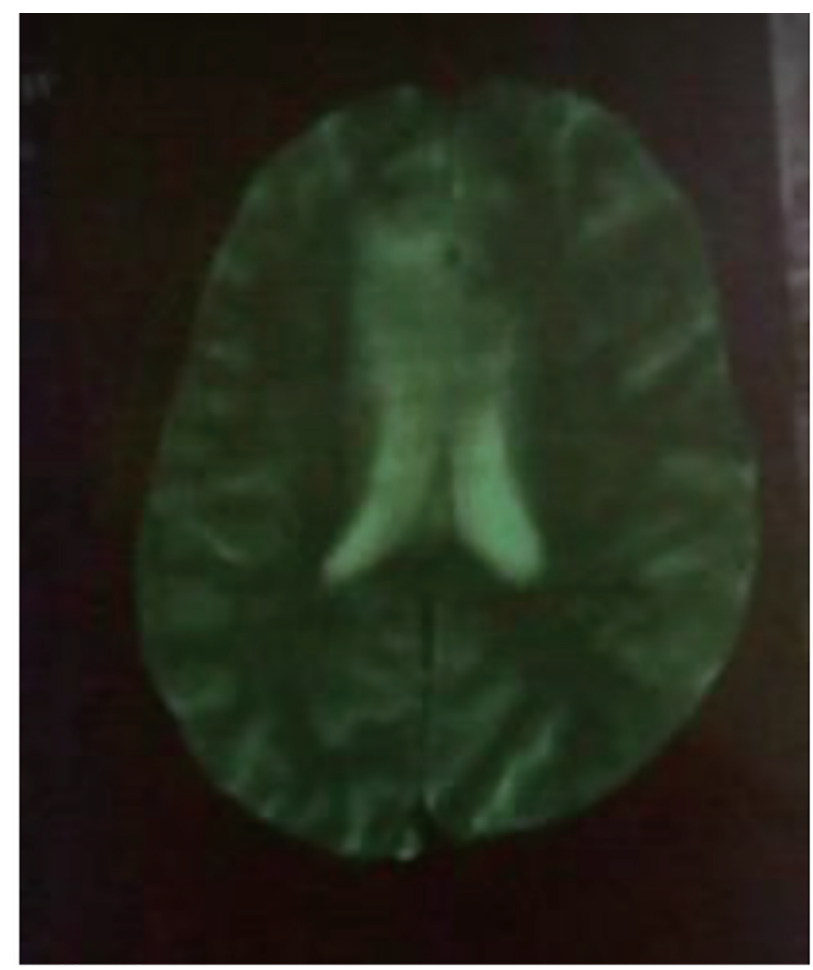

Figure 3. MRI: cerebral axial section through the ventricles moderate enhancement especially at the knee of the corpus callosum and in the right oval center.

described and the appearance of a new range with the same characteristic of the first one in parieto-temporo-occipital left area. This lesion concerns the white sub-cortical substance (Ushaped fibers in parieto-temporo-occipital left area reaching the left corpus callosum). It is in hyposignal T1 and hypersignal T2 with a moderate peripheral enhancement after injection
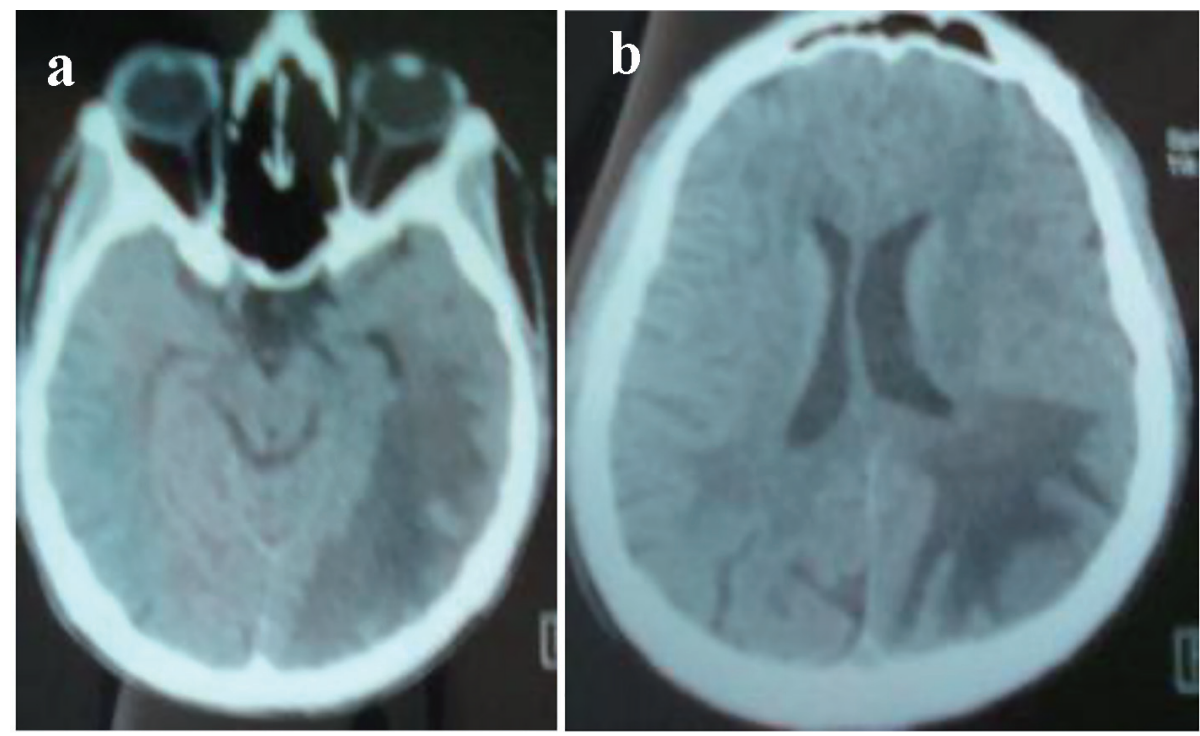

Figure 4. $(\mathrm{a}, \mathrm{b})$ Brain $\mathrm{CT}$ : hypodensity in left cortico and under cortical parietooccipital area and in the white matter of the right centrum semiovale. 

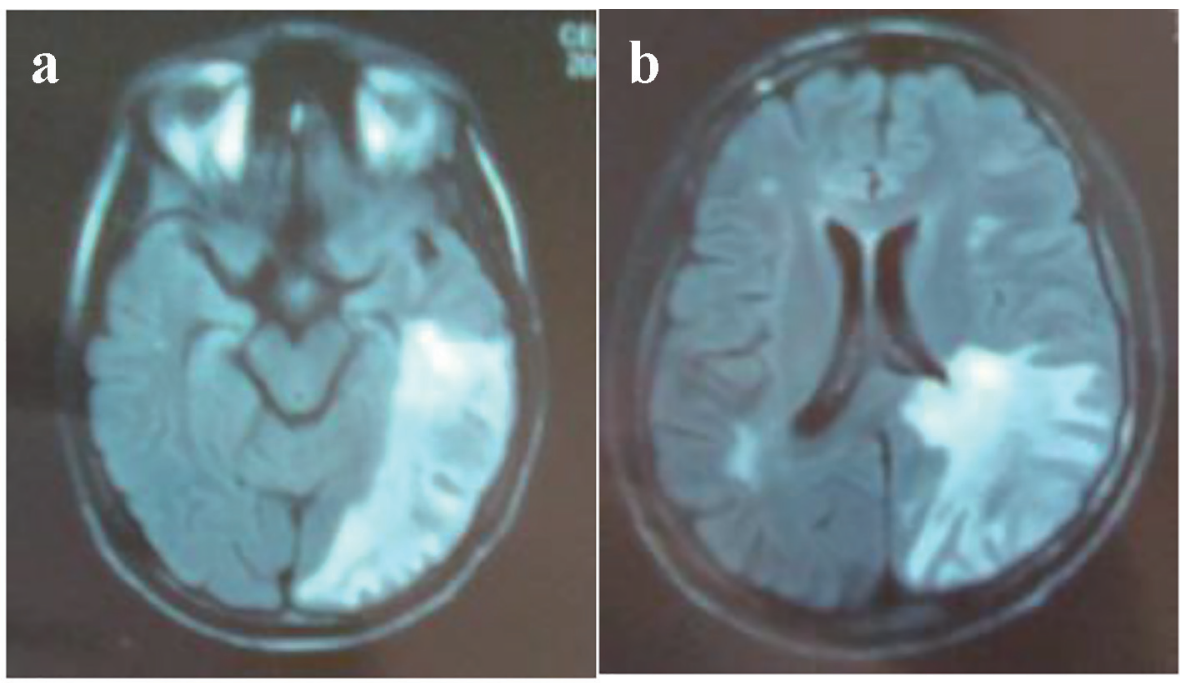

Figure 5. (a, b) MRI: cerebral axial section: hypersignal T2 in parieto-temporo-occipital left area reaching the left corpus callosum lesions.

of gadolinium and discrete mass effect on the occipital horn of the lateral homolateral ventricle without mass effect on the median line (Figs. 5 and 6).

\section{Discussion}

In the current case report, we describe a patient in whom PML developed during chronic PD over 4 years of dialysis therapy. The diagnosis of PML was strongly suggested by clinical symptoms and brain MRI. No similar cases have been reported in the literature. Indeed, the only reported cases occurred in hemodialytic cases. Three cases from 40 hemodialytic patients were reported in a recent study by Kamar et al. They were treated by interferon and erythropoietin for hepatitis $\mathrm{C}$ and returned in remission after the stop of interferon [5].

The two other cases, in hemodialysis, were reported in 1984 and 2006. The one reported in 1984 was the first case described. It was a 56-year-old Japanese man who received prolonged hemodialysis treatment over 11 years, and his outcome was fatal, fell into a deep coma and quickly died [6]. The second case was reported in 2006. It was a 57-year-old male who developed PML after HTLV-1 infection. Rearranged regulatory region of JCV DNA was detected. The two precipitating factors in this case apart the hemodialysis were a liver cirrhosis induced by hepatitis C and HTLV-1 infection [4].

Usually, in immunocompromised patients, the beginning is subacute and deficits are immediately serious. The most common symptoms are motor weakness and cognitive disorders $(50 \%$ of cases). Other signs are aphasia, visual (visual field or oculomotor paralysis), sensory, and headache ( $15 \%$ of cases). Epileptic seizures are less frequent and occur in $10 \%$ of patients [7]. In our case, only motor system was affected with behavioral disorders in the first episode and in the second episode, cognitive disorders especially memory and visual field were affected.

CT scan shows hypodense lesions in white substance.
There is no mass effect or of enhancement [7]. MRI brain shows lesions predominant in semi-oval centers and the frontal, parietal, and occipital lobes. It is in accord with our case since cerebral lesions were especially located in frontal, cortical and subparietal white matter, right centrum semiovale knee and corpus callosum.

The enhancement of lesions on MRI would be in relation with a local inflammatory reaction. This local reaction explained by a restoration of immunity causes a break of bloodbrain barrier that induces the enhancement in imaging. The en-

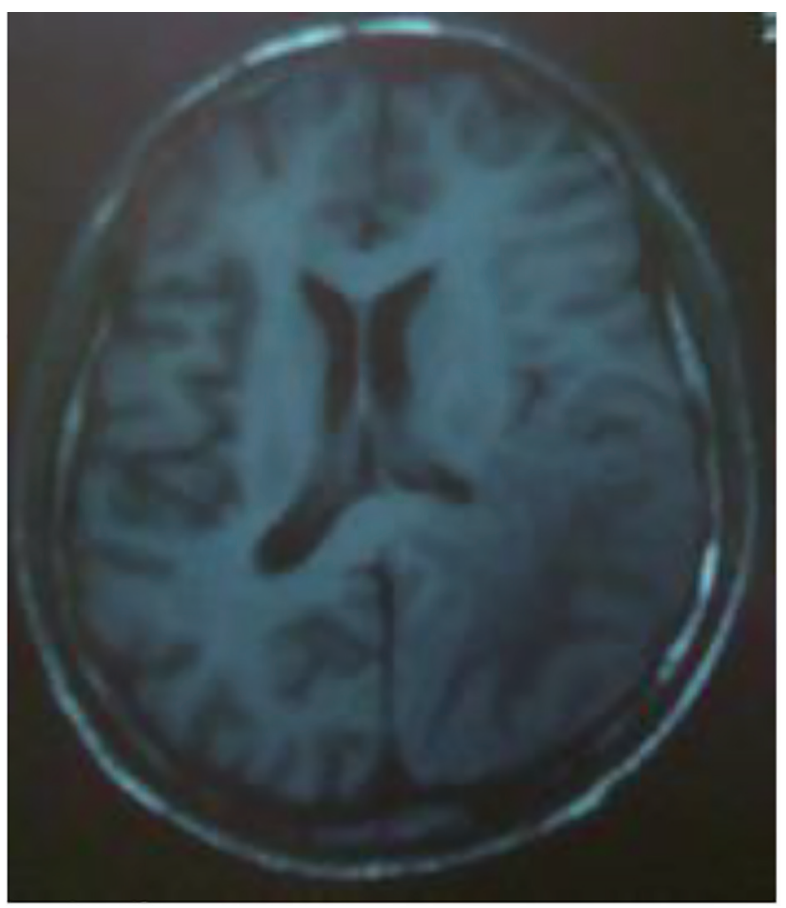

Figure 6. MRI: cerebral axial section: hyposignal T1 in parieto-temporo-occipital left area reaching the left corpus callosum lesions. 
hancement of lesions and cortical involvement were atypical but referred to a good prognosis.

Lumbar puncture is very important in eliminating differential diagnosis. Usually, there are less than 20 cells $/ \mathrm{mm}^{3}$. A major protein level $(>2 \mathrm{~g} / \mathrm{L})$ is found in more than half of the cases. The hypoglycorrhachia is observed in less than $15 \%$ of patients [7]. However, the diagnosis key is the detection of JCV. It has a high specificity (96-100\%) and sensitivity average (50-75\%). A high rate of DNA JC V found in CSF is a bad prognostic factor for patient survival [7]. This analysis is much less invasive than histological evidence which remains, however, a reference for the diagnosis. Its sensitivity ranges from $64 \%$ to $96 \%$ and specificity is $100 \%$.

PCR on biopsy tissue virus confirms the diagnosis. Diagnosis is made through a clinical, radiological and biological (PCR positive CSF) arguments. Diagnostic criteria have been recently established.

PML is "histologically confirmed" (viral DNA detection by PCR on biopsy tissue), or "biologically confirmed" (detection of viral DNA by PCR CSF), or "possible" (clinical aspects and radiological supports despite a negative PCR) as our case represents [8].

It is well recognized that PML requires an immunosuppressed state for a florid expression of this disorder. As occurred in our case, chronic dialysis, hepatic dysfunction and myelodysplasia are all compromised elements and constitute an immunodepressed status.

Most immunosuppressive states were associated to PML including AIDS, lymphoproliferative disorders, such as leukemia and lymphoma, systemic diseases, organ transplantation, cirrhosis, immunosuppressive agents, immunodeficiency of genetic origin [4]. This deranged cell-mediated immunity (CD4 and CD8 lymphocytes) would expose PML. Myelodysplasia in our case belongs to these states of immunosuppression and induces such disturbances in immune system.

The elements of favorable prognosis in our case are the negativity of PCR JCV in the CSF and the enhancement in control imaging which is a good indicator of immunity restoration. The element of bad prognosis is the presence of mass effect in MRI.

\section{Conclusion}

In conclusion, in the present paper, we have reported a case manifesting the development of PML in patient undergoing PD with myelodysplasia associated to liver dysfunction. The precipitating causes appear multifactorial, but the depressed immune system induced by chronic PD therapy constitutes a determinant of possible development of PML.

Cayeux et al reported that this immunodeficient state is due in part to a defect in IL-2 production [9]. The use of low dose IL-2 had successfully induced remission after marrow transplantation. In our case, only recurrent plasma exchanges induced total recover.

\section{References}

1. Sudhakar P, et al. Progressive multifocal encephalopathy:recent progress and neuro-ophtalmology. Journal Neuro Ophthalmology. 2015;35(3):296-305.

2. Rueger MA, Miletic H, Dorries K, Wyen C, Eggers C, Deckert M, Faetkenheuer G, et al. Long-term remission in progressive multifocal leukoencephalopathy caused by idiopathic CD4+ T lymphocytopenia: a case report. Clin Infect Dis. 2006;42(7):e53-56.

3. Alibert S, et al. Progressive multifocal encephalopathy due to $\mathrm{JC}$ virus during a treatment of hepatitis $\mathrm{C}$ after renal transplantation. Gastroenterol Clin Boil. 2006;30:473475.

4. Matsuda H, Hayashi K, Meguro M, Saruta T. A case report of progressive multifocal leukoencephalopathy in a human T-cell lymphotropic virus type 1-infected hemodialytic patient. Ther Apher Dial. 2006;10(3):291-295.

5. Kaamar N, et al. Reversible posterior leucoencephalopathy syndrome in hepatitis $\mathrm{C}$ virus positive long term hemodialysis patients. Am J kidney Dis. 2001;37:E24.

6. Matsubara $\mathrm{O}$, et al. Progressive multifocal encephalopathy associated with prolonged hemodialysis treatment. Virchows Arch A Pathol Anat Histopathol. 1984;403(3):301305.

7. Brunot S, et al. Progressive multifocal leukoencephalopathy in an immunocompetent man. The Letter of Neurologist. 2011;15(6):227-228.

8. Cinque P, Koralnik IJ, Clifford DB. The evolving face of human immunodeficiency virus-related progressive multifocal leukoencephalopathy: defining a consensus terminology. J Neurovirol. 2003;9(Suppl 1):88-92.

9. Przepiorka D, Jaeckle KA, Birdwell RR, Fuller GN, Kumar AJ, Huh YO, McCutcheon I. Successful treatment of progressive multifocal leukoencephalopathy with low-dose interleukin-2. Bone Marrow Transplant. 1997;20(11):983-987. 